OAl-PMH: http://www.indteca.com/ojs/index.php/Revista Scientific/oai

Artículo Original / Original Article

\title{
La responsabilidad ambiental de la gerencia de gestión ambiental de la Municipalidad Provincial del Santa
}

\author{
Autora: Evelyn Marcia Urquiaga Juárez \\ Universidad Privada Antenor Orrego, UPAO \\ eviurqui@hotmail.com \\ Chimbote, Perú \\ https://orcid.org/0000-0001-7775-6234
}

\section{Resumen}

El presente estudio tuvo como objetivo general; determinar los factores que ocasionan la ineficacia de la normatividad de responsabilidad ambiental para ejercer el control de la protección del medio ambiente por parte de la Municipalidad Provincial del Santa. Además, se utilizó un tipo de investigación de acuerdo con la naturaleza; fue una investigación cuantitativa, porque brindó una descripción completa y detallada del tema de investigación. La unidad de análisis fue a través de registros de proyectos efectuados por la sub-Gerencia de Gestión Ambiental de la Municipalidad Provincial Del Santa, para lo cual se elaboró una guía de observación que tuvo la validez del juicio de expertos para la recopilación documental. El estudio concluyó que la ineficacia en la aplicación de la normatividad de la responsabilidad ambiental trae consigo consecuencias irreparables al medio ambiente, los daños que se originan son irremediables y se vuelve imposible la recuperación, además la ineficacia en el control de la normatividad provoca que la contaminación tenga efectos negativos en la salud de las personas y atenta contra el derecho de la salud y más aun provocando que no se cumpla la Ley del medio ambiente.

Palabra clave: medio ambiente; gestión ambiental; contaminación. Código de clasificación internacional: 5605.02 - Derecho civil.

Cómo citar este artículo:

Urquiaga, E. (2021). La responsabilidad ambiental de la gerencia de gestión ambiental de la Municipalidad Provincial del Santa. Revista Scientific, 6(21), 180-200, e-ISSN: 2542-2987. Recuperado de: https://doi.org/10.29394/Scientific.issn.2542-2987.2021.6.21.9.180-200

Fecha de Recepción: 10-04-2021
Fecha de Aceptación: 03-07-2021
Fecha de Publicación: 05-08-2021 
OAl-PMH: http://www.indteca.com/ojs/index.php/Revista Scientific/oai

Artículo Original / Original Article

\title{
The environmental responsibility of the environmental management department of the Provincial Municipality of Santa Cruz
}

\begin{abstract}
The present study had as a general objective; determine the factors that cause the ineffectiveness of the environmental responsibility regulations to exercise control of the protection of the environment by the Provincial Municipality of Santa. In addition, a type of investigation according to nature was used; it was a quantitative investigation, because it provided a complete and detailed description of the research topic. The unit of analysis was through project records made by the Sub-Management of Environmental Management of the Provincial Municipality of Santa, for which an observation guide was prepared that had the validity of the expert judgment for the documentary compilation. The study concluded that the ineffectiveness in the application of the environmental responsibility regulations brings with it irreparable consequences to the environment, the damages that originate are irremediable and recovery becomes impossible, in addition the ineffectiveness in the control of the regulations causes that the Pollution has negative effects on people's health and violates the right to health and even more so by not complying with the Environmental Law.
\end{abstract}

Keywords: environment; environmental management; contamination. International classification code: 5605.02 - Civil law.

\footnotetext{
How to cite this article:

Urquiaga, E. (2021). The environmental responsibility of the environmental management department of the Provincial Municipality of Santa Cruz. Revista Scientific, 6(21), 180-200, eISSN: 2542-2987. Recovered from: https://doi.org/10.29394/Scientific.issn.25422987.2021.6.21.9.180-200
}

Date Received: 10-04-2021
Date Acceptance:

03-07-2021
Date Publication: 05-08-2021 


\section{Introducción}

La responsabilidad ambiental, es el cuidado y el amor al lugar donde vivimos, se enfoca en diferentes acciones que benefician a la comunidad, donde prima la disminución de problemas o necesidades ambientales, de tal forma que la vida de la población mejora enormemente. La responsabilidad ambiental tiene un amplio margen para conocer y desarrollar todas sus dimensiones que contribuye a beneficiar a la sociedad, cabe resaltar que estas acciones recaen tanto en las personas, como en las organizaciones y más aún en el estado, que a través de sus normativas debe supervisar y cuidar la salud y la vida de la población.

El Perú, a través del Ministerio del Ambiente (MINAM) no está siendo responsable en el cuidado al medio ambiente y esto afecta negativamente al ecosistema, así mismo; los gobiernos regionales o provinciales no están ejerciendo un control para reducir la contaminación, en el caso de Madre de Dios y Puno donde la contaminación por mercurio alcanza altos niveles en el ecosistema como en seres humanos.

En la ciudad de Chimbote, se presentan innumerables problemas ambientales, como la contaminación de la bahía el Ferrol provocadas por inescrupulosas empresas pesqueras que tiran sus desechos a la bahía de forma ilegal, la contaminación de residuos sólidos debido al incremento de la población en distintas zonas de la provincia y la emisión de desechos en las calles, la contaminación del aire a través de los desechos tóxicos que se emanan de las fábricas.

La ineficacia en la aplicación de las normativas ambientales a través de la Sub Gerencia del Ambiente de la Municipalidad Provincial del Santa, no está cumpliendo con sus funciones, esto ha provocado en la ciudad playas contaminadas, una mínima existencia de áreas verdes y las que existen están desapareciendo paulatinamente, las principales calles están llenas de basura y los residuos sólidos no se reciclan debidamente, la contaminación del aire y 
los desechos que emanan las fábricas hacia el mar son algunas causas que tienen efecto negativos en el medio ambiente.

Existen estudios que se presentan como antecedentes para profundizar más en el tema; las investigaciones internacionales. Para Costa, Santos y Ángelo (2020): quienes elaboraron su artículo científico sobre la responsabilidad social y medioambiental empresarial, para saber si los consumidores la perciben como un valor organizativo, en ese sentido el objetivo fue investigar si los consumidores perciben la responsabilidad social y ambiental corporativa como un valor organizacional adquirido o como existente debido a otros factores.

Como conclusión del estudio, se concluyó que los consumidores no perciben la adopción de SER por parte de las empresas como un valor adquirido sino como una estrategia corporativa, es decir, como vinculada a otros intereses. Así, los consumidores dejan claro que sí creen que SER es un valor adoptado por las empresas.

Mencionando a Collantes y Giraldo (2020): realizaron su investigación sobre la responsabilidad ambiental como estrategia competitiva de las organizaciones, tuvo como objetivo general conocer si las estrategias empleadas les traen reconocimientos sociales. Finalmente, las empresas al realizar una responsabilidad ambiental, las acciones son las que generan una buena relación con la sociedad.

Acorde con esto, las organizaciones con la buena práctica mejoran su imagen y reputación, además que la sociedad y las personas las reconocen por las buenas prácticas. Estas acciones también son manejadas como tácticas o estrategias con el único propósito de crear competitividad el cual les permita tener una mejor sostenibilidad y una larga vida, ante los controles y supervisiones de los entes encargados. Últimamente, la responsabilidad de las organizaciones como estrategia competitiva, tiene relación con la calidad de productos que comercializa, las acciones en frente del medio ambiente y que 
la población observa, todo el conglomerado puede fortalecer su reputación corporativa.

Para el autor Femenías (2017): quien desarrollo su artículo sobre cómo se relaciona la responsabilidad por un daño ambiental con el sistema de evaluación del impacto ambiental. El estudio concluye; que existen actividades o acciones que generan un impacto negativo en el medio ambiente y todas las acciones negativas deben ser ingresadas al Sistema de Evaluación de Impacto Ambiental (SEIA), sin embargo, no lo hicieron.

De tal forma, la responsabilidad por un daño ambiental producido, según el Ministerio Secretaría General de la Presidencia (1994): tendría como consecuencia legal un incumplimiento que se observa en la situación prevista del inciso $1^{\circ}$ del art. 53 de la Ley sobre Las Bases Generales del Medio Ambiente - Ley 19300, esto porque se encontraría infringiendo la norma en el cuidado del medio ambiente establecida en la misma ley. Por otro lado, están las actividades o acciones que generan un impacto negativo en el medio ambiente y todas las acciones negativas deben ser ingresadas al SEIA, si lo hicieron.

En el análisis de los factores que provocaron un daño al medio ambiente, se debe conocer primero si son daños que están dentro del límite permitido, daños que no están dentro del límite permitido o daños que están fuera de ese contexto. Si fuera de daños que no están en el contexto, se debe analizar si son daños reparables o irreparables.

También existen antecedentes nacionales, que aportan al trabajo de investigación como de Bustamante y León (2015): quienes elaboraron su tesis sobre la normativa ambiental en el Perú con la gestión de los residuos sólidos que emana de las construcciones y demoliciones como efecto de las excavaciones en todas las edificaciones.

La investigación concluyó que, la normativa del Ministerio de Vivienda, Construcción y Saneamiento (MVCS, 2013a): a través del Decreto Supremo 
N. ${ }^{\circ}$ 003-2013-VIVIENDA, que para el Congreso de la República (2000): no tiene los correctos criterios en sus clasificaciones de los materiales en comparación con la Ley N. ${ }^{\circ} 27314$ - Ley General de Residuos Sólidos (24.07.04) en el Decreto Supremo N. ${ }^{\circ}$ 057-2004-PCM de la Presidencia del Consejo de Ministros (PCM, 2004), y esto se debe porque la normativa del Decreto Supremo N. ${ }^{\circ}$ 003-2013-VIVIENDA está por debajo de ellos. En la Ley N. ${ }^{\circ} 27314$ del año 2004, existen mejores criterios fundamentados técnicamente, los cuales se respaldan con los análisis de los estudios de suelos antes de realizar cualquier trabajo.

Cabe precisar que la normativa ambiental Decreto Supremo N. ${ }^{\circ} 003-$ 2013-VIVIENDA del Ministerio de Vivienda, Construcción y Saneamiento (MVCS, 2013b): exterioriza que la gestión de los residuos sólidos es una consecuencia de una construcción o demolición y resto apunta como efecto a los residuos ocasionados en la fase de construcción y acabados del edificio. Además, la poca mano de obra instalada para el manejo de todos los residuos sólidos es insuficiente y afecta negativamente a la productividad, lo cual tiene un origen en la poca flota de las Entidades Prestadoras de Salud de Residuos Sólidos [EPS-RS] que se planificó en las cotizaciones en un inicio, en donde solo indica 041 unidades de volquetes disponibles como máximo, en el caso práctico.

En las investigaciones locales, García (2017): con su tesis sobre las existentes políticas públicas en beneficio del medio ambiente y los efectos negativos con la contaminación ambiental que existe en el mercado La Paradita del centro poblado San Jacinto.

El estudio concluyó, que en todas las partes del mundo existe la contaminación y que esto afecta tanto al medio ambiente como a las personas, de tal forma; las personas que laboran en la Paradita que es el mercado de la localidad de San Jacinto están a diario expuestos a contagiarse de diversas enfermedades por la contaminación que está en dicho establecimiento. 
Para sumar a la problemática, las causas existentes que ayudan a la contaminación son los desechos que arrojan las personas a las calles, los desperdicios que se arrojan del mercado, los residuos que se emana de la agricultura y ganadería, el tráfico vehicular que existe por los mototaxistas y el ruido que provocan, existen diversas causas pero la principal causa de la contaminación es la falta de responsabilidad y conciencia de mejorar su ciudad y el medio ambiente por parte de los habitantes y las autoridades.

Adicionalmente, Rojas (2017): realizó su trabajo de investigación sobre la eficiencia "de las Normas de Gestión ambiental en empresas pesqueras y describir los problemas de salud de los pobladores de Coishco viejo" (pág. 7). El estudio concluye que no cumplen eficazmente las normas sobre la gestión ambiental, esto se origina por la ineficiente supervisión del cumplimiento de las normas o leyes que debe realizar la comuna edil o responsables del cuidado del medio ambiente.

Existen diversas instituciones u organizaciones públicas y privadas que son las responsables de supervisar el cumplimiento de las normas como el Ministerio del Ambiente, La Comuna de Coishco y Sub-Gerencia del Medio Ambiente y el Ministerio Público,

Sin embargo, la industria pesquera en la localidad de Coishco Viejo, no trabajan responsablemente en el cumplimiento de las normas y esto se ve reflejado en los afluentes que arrojan y que contamina el mar de la ciudad, originando enfermedades que perjudican la salud de los pobladores como alergias en los niños y ancianos e irritación en los ojos por el humo de las fábricas y en casos fortuitos problemas respiratorios que han sido de nivel hospitalario, esto se refleja en los índices que presenta EsSalud en Perú, entre los años 2011-2017. Finalmente, la contaminación del aire, el suelo y mar son los causantes de problemas de asma, manchas en la piel irritación, infección en las vías respiratorias hasta pueden provocar cáncer. 


\subsection{Las teorías relacionadas al tema}

El concepto de responsabilidad ambiental, conforme a Enger y Smith (2006): quienes manifiestan que el ambiente debe tener el merecido respeto moral y no la que se deriva nada más por intereses personales o externas. Apunta que el ambiente debe mantener sus derechos directos que se aprecian como cubierta moral digna de un deber directo.

Para Gardetti (2004): la responsabilidad ambiental tiene como base la ética y la operatividad, que se fundamenta con la ética de compensación y adeudo que se induce de diferente manera al negociar, originar, gastar y referirse a las instituciones en su contexto global. Además, reconoce todas las insuficiencias "operativas" de los distintos entes del estado y las organizaciones privadas, enunciadas en la investigación de una mayor creación y (Eco) eficiencia convertida en el mejor logro en el desempeño de todas sus funciones encomendadas. Por ejemplo, las organizaciones deben ser sostenibles en un largo plazo, el ente fiscalizador debe ser eficiente en el desarrollo laboral, en el caso de las buenas prácticas de las organizaciones sin fines de lucro, estas logran fortificar su sostenibilidad y desarrollo institucional.

Según el bien jurídico hace referencia al derecho ambiental y lo que significa la conservación de todo el medio ambiente, la interpretación de Cafferatta (2004): enseña que el derecho ambiental, en el método jurídico es el conjunto de normas reguladoras en relación al derecho público o privado, tendiendo en consideración disciplinaria todas las conductas en lista según el ordenamiento pensante y la conservación de todo el medio ambiente, además de prevenir algún daño el mismo que a fin de alcanzar el sustento equilibrado natural, lo que excederá en una mejoría en la calidad de vida y del ambiente.

Para Dehays, Baca y Bosker (2000): el medio ambiente se define como la agrupación de complementos del medio natural como la selva, la flora, la fauna, el suelo, la tierra, el aire, el clima, los ríos, el océano, el agua, y su 
relación. En referencia al tema, Andía (2013): manifiesta que es la integración de los elementos creados por el hombre y la naturaleza, para el desarrollo y la existencia de la humanidad y la preservación de la vida, además de la relación entre los seres vivos y la relación de la naturaleza con el hombre.

En consonancia con Romero, Diego y Álvarez (2006): el daño ambiental es la muerte de todos los elementos que conforman el ecosistema, como pueden ser las alteraciones contaminantes del aire, el agua, en muchos casos son el resultado de aquellas actividades de la persona que contaminan el medio ambiente. La Gestión Ambiental, en la declaración de Río adoptada en el marco de las Naciones Unidas sobre medio ambiente y desarrollo, los estados se comprometen a cuidar que las actividades desarrolladas en sus territorios no causen daños a otros estados, fortalecer sus capacidades para el desarrollo sustentable, facilitar la participación ciudadana en la gestión ambiental, permitir un acceso efectivo a las actividades administrativas y judiciales para reclamaciones ambientales, etc.

Asimismo, Quisocala (2003): afirma que el cumplimiento de estas responsabilidades ambientales implica la ejecución de una serie de actividades encaminadas a promover una ordenación del medio ambiente y a contribuir al modelo de desarrollo sustentable. Cualquier elemento negativo químico o biológico que está presente en el medio ambiente origina una contaminación que afecta y pone en riesgo la salud de las personas y el bienestar de la sociedad.

\section{Metodología}

En la presente investigación se aplicó el método científico o hipotético deductivo, fue una investigación cuantitativa y tuvo un diseño no experimental con una sola variable que no fue manipulada y la ejecución fue en un tiempo determinado. El estudio fue de tipo descriptivo, en donde el investigador describió toda la información sobre la variable en estudio en su forma natural, 
describiendo características, sucesos y cualquier clase de información propia del estudio, para su análisis posterior. Se utilizó la técnica del análisis de documento y como instrumento la guía de análisis de documental.

La población fue toda la información o registros de proyectos analizados tanto internacionales, nacionales y en los locales los que fueron efectuados por la sub-Gerencia de Gestión Ambiental de la Municipalidad Provincial Del Santa entre los años 2015 y 2018, en su totalidad se registra 10 expedientes técnicos. La muestra estuvo constituida por los 10 expedientes técnicos, a este tipo de muestra se le conoce como muestra censal o muestra poblacional. Es decir; no es necesario utilizar algún tipo de formula porque se está tomando el número total de la población.

\section{Resultados}

En este capítulo se presentan los proyectos según la normatividad extranjera, nacional y local, de los cuales se han escogido proyectos con bases en la normativa vigente de cada lugar, con relevancia en el cuidado del medio ambiente y de gran importancia para la salud, son proyectos actuales con efectos positivos que se han visto reflejados.

\subsection{Proyectos internacionales}

Proyecto plan aire, desarrollado en España en el año 2016, el Parlamento Europeo puso en marcha una campaña en contra de la contaminación ambiental, denominado Programa Nacional de control de la contaminación atmosférica, cumpliendo con la normativa española (UE) 2016/2284. El programa se contempla en la disminución de la contaminación a manos de las emisiones de las empresas que contaminan la atmosfera, para el caso se ha modificado la norma 2003/35/CE y se elimina la norma 2001/81/CE.

Las grandes industrias, el parque motor, los desechos contaminantes 
son algunos factores que ha provocado que el estado español inicie el programa con estrategias para mejorar la calidad del aire en todo el país, principalmente se debe combatir para disminuir las emisiones de dióxido de nitrógeno $\left(\mathrm{NO}_{2}\right)$, ozono $\left(\mathrm{O}_{3}\right)$, dióxido de azufre $\left(\mathrm{SO}_{2}\right)$, partículas PM10 y partículas PM2,5.

Proyecto coche eléctrico, en la edición de Mohorte (2018): se muestra que Noruega es el único país del mundo que está dejando de lado los coches convencionales a base de combustible y se está trasladando a los coches eléctricos, así mismo respirar en Noruega es más sano que nunca debido que el $60 \%$ del parque automotor es eléctrico, claro ejemplo es Bergen la segunda ciudad más importante de Noruega en el cual los niveles de contaminación del aire ha descendido y se debe a que la emisión del dióxido de nitrógeno se ha reducido un $14 \%$ desde el 2002 . El tráfico ha disminuido un $6 \%$ entre los años 2015 y 2017, además que en la actualidad recorren hoy un $20 \%$ menos autos en sus avenidas que hace dos décadas.

Proyecto captura y almacenamiento de carbono, Noruega está desarrollando un programa en la captura y almacenamiento del carbono para ello ejecutará infraestructura adecuada para poder minimizar hasta el 2022 las emisiones del $\mathrm{CO}_{2}$ al medio ambiente. Noruega planea capturar las emisiones de carbono y transportarlo a las reservas vacías de petróleo, este proyecto tendrá una inversión entre 420 y 860 millones de euros.

Si Noruega logra desarrollar este proyecto innovador y llega ser un éxito podría ayudar a minimizar la emisión de dióxido de carbono a nivel mundial, según la Agencia Internacional de Energía (AIE) el desarrollo de la técnica de captura y almacenamiento del carbono es un factor determinante para reducir las emisiones de dióxido de carbono, pero existen países que han rechazado estas técnicas debido al alto coste de inversión. 


\subsection{Proyectos nacionales}

Proyecto planta de tratamiento de residuos sólidos, desarrollado en la ciudad de Trujillo en donde existe una serie de problemas ambientales, desde los desechos sólidos arrojados en la vía pública, el botadero ilegal del milagro. En ese sentido; para resolver los problemas, la comuna trujillana realizó una serie de petitorios al Ministerio del Ambiente para que pueda dar la confianza a la empresa privada Intevas C6 Energy Services y se logre la construcción de la planta de tratamiento, donde la comuna norteña no invertirá nada todo será responsabilidad de la empresa privada.

La cual tendrá una concesión por 25 años, para la reducción de los desechos el tratamiento contemplará residuos sólidos de construcción, neumáticos, desechos hospitalarios, domésticos, plásticos y vidrios, con todo esto se logrará obtener nuevos productos reutilizables. Se tiene planeado eliminar el botadero el Milagro y reducir la contaminación urbana.

Plan Nacional para reducir contaminantes climáticos, el Perú está dentro de los cincuenta países más contaminados del planeta con altos índices de contaminación del aire por las industrias pesqueras, mineras o siderúrgicas las cuales no son supervisadas adecuadamente en la emisión del $\mathrm{CO}_{2}$, de tal manera la Coalición Clima y Aire Limpio (CCAC) en conjunto con la ONU forma la intervención internacional de la ONU trabajaron en un programa nacional para disminuir las sustancias contaminantes de la vida corta, para ello, se estima que ayudará a eliminar toda la contaminación que proviene del carbono negro, metano y ozono, con el fin de disminuir el cambio climático.

El proyecto tendrá un coste de inversión de 250 mil dólares americanos, para esto se tuvo programado en una primera fase la construcción de un área especial para el control de las emisiones.

\subsection{Proyectos locales}

Proyecto para el Estudio de Pre-Inversión en la Planta de 


\title{
Tratamiento de Agua Residual para Chimbote y Nuevo Chimbote (PTAR-
}

\author{
2), este proyecto se desarrolla en Chimbote por su crecimiento poblacional ha
} provocado que el sistema de drenaje colapse y que el punto de desembocadero sea la bahía. La contaminación que sufre directamente la bahía a manos de los desechos domésticos ha tenido efectos negativos en toda la costa y ningún gobierno de turno ha podido solucionar.

De tal manera, gracias a un fondo alemán se está realizando un estudio de pre-inversión para la construcción de una planta de tratamiento de aguas residuales domesticas para Chimbote y Nuevo Chimbote, es así que cuando la obra se concrete dejara de arrojar sus desechos en el mar. Este proyecto será financiado gracias a una cooperación alemana y el estado peruano a través del Ministerio de Economía y Finanzas (MEF, 2018): en Resolución Suprema N. ${ }^{\circ}$ 025-2018-EF. Por lo tanto, ante la problemática existente se vio la necesidad de empezar con un megaproyecto como PTAR-2, para disminuir la contaminación en la costa de Chimbote, para beneficiar miles de ciudadanos.

Tabla 1. Planta de tratamiento de agua Residual.

\begin{tabular}{|c|c|}
\hline $\begin{array}{c}\text { Nombre del } \\
\text { proyecto }\end{array}$ & $\begin{array}{c}\text { Planta de Tratamiento de Agua Residual para Chimbote y } \\
\text { Nuevo Chimbote PTAR-2 }\end{array}$ \\
\hline $\begin{array}{c}\text { Objetivo del } \\
\text { proyecto }\end{array}$ & $\begin{array}{l}\text { El fin del proyecto es construir una planta de tratamiento de } \\
\text { aguas residuales domesticas para Chimbote y Nuevo Chimbote } \\
\text { para no arrojar sus desechos en el mar. }\end{array}$ \\
\hline
\end{tabular}

\begin{tabular}{|c|l|}
\hline \multicolumn{2}{|c|}{ Unidad formuladora y ejecutora del proyecto de inversión pública } \\
\hline Sector & Gobierno Local \\
\hline Pliego & Municipalidad Provincial Del Santa \\
\hline Entidad & Ministerio de Vivienda, Construcción y Saneamiento (MVCS) \\
\hline Consultora & CES Consulting Engineers Salzgitter Gmbh (Alemania) \\
\hline Inversión & Euros 1'070,733.030 - S/ 4'146,520 Soles \\
\hline Tiempo & 04 años \\
\hline Beneficiarios & Chimbote (334,568 hab.) - Nuevo Chimbote (159,321 hab.) \\
\hline
\end{tabular}

Fuente: La Autora (2021). 
Se refleja en la tabla 1 , el proyecto de la planta de tratamiento de agua residual apunta a disminuir y terminar con la contaminación que aún existe en el litoral, debido al desemboque de los derechos domésticos que terminan en la playa, al mismo tiempo, beneficiará a toda la población de los distritos de Chimbote y Nuevo Chimbote con la mejora de las líneas del sistema de drenaje con una inversión de 1'070,733.030 de euros, solamente para el estudio de prefactibilidad, para el desarrollo de la misma se tendrá otro presupuesto mayor. En ese marco, la gerencia de gestión ambiental de la Municipalidad Provincial del Santa está a cargo de la supervisión y en coordinación con la empresa alemana CES Consulting Engineers Salzgitter Gmbh, que viene trabajando arduamente para que el proyecto sea una realidad. Una vez logrado, se iniciará con el desarrollo con el único fin que todos los desechos domésticos desemboquen a una distancia apropiada lejos de la orilla del mar.

Proyecto de limpieza pública con la compra de compactadoras, en Chimbote existe un déficit en la parte operativa en el servicio de recolección de residuos sólidos. Sin embargo, la gerencia de limpieza pública de la Municipalidad Provincial del Santa está realizando una seria de actividades para mejorar el recojo de residuos sólidos a través del ordenamiento jurídico, como se lo redacta el Congreso de la República (2003): en la Ley N. ${ }^{\circ} 27972$ Ley Orgánica de Municipalidades.

De esta forma, se está aprobando la compra de diversos activos para mejorar el recojo de basura de las distintas zonas de la ciudad que sufren de esta problemática, es así como la compra de compactadoras, barredoras mecánicas, contenedores y motocargas de basura, entre otros activos para apoyar la disminución de la contaminación a través de los desechos.

Proyecto construcción de una planta de tratamiento de residuos, el problema en Chimbote es la existencia de botaderos clandestinos de residuos sólidos lo cual ha traído efectos negativos a la salud con enfermedades respiratorias, además de provocar incendios, malos olores y 
criadero de roedores.

Para contrarrestar, se está realizando un proyecto para la construcción de una planta de tratamiento de residuos sólidos y el recojo de basura, para que la basura no se pierna enterrada en el suelo porque sería perjudicial para la naturaleza, de tal manera se contará con tecnología que hará que la materia orgánica e inorgánica se industrialice. A través de la Ley N ${ }^{\circ} 27446$ - Ley del Sistema Nacional de Evaluación de Impacto Ambiental y su Reglamento del Ministerio del Ambiente (2011): con esta construcción se logrará disminuir los botaderos clandestinos o los puntos de acopio de reciclaje informal para convertirla en una ciudad más limpia y sostenible, respetando el medio ambiente.

Proyecto Apro Chimbote, el mar de Chimbote es ahora un mar oscuro donde toda clase de desperdicio se arroja de forma irresponsable esto ha hecho que el nivel de contaminación de la había sea alta y muy difícil de limpiar. Es así que en el gobierno del ex presidente Alejandro Toledo, se firmó el Decreto Supremo N. ${ }^{\circ}$ 005-2002-PE del Ministerio del Ambiente (2012): con el fin de crear una comisión técnica multisectorial por los problemas de contaminación a la Bahía El Ferrol y declararlos de interés nacional, por lo tanto, en el año 2012 se aprobó la Resolución Suprema N. ${ }^{\circ}$ 004-2012-MINAM con el propósito de diseñar un Plan para la Rehabilitación Ambiental, este plan se basa en la construcción de afluentes para el sector pesquero para cumplir con los límites máximos permisibles (LMP) y su desembocadura a externas de la bahía.

El proyecto se realizó y se puso en marcha en el año 2015 y hasta la fecha ya tiene casi el $95 \%$ de todos los afluentes de la ciudad y se espera en un corto tiempo llegar al $100 \%$ del sector.

Proyecto Parque Metropolitano de Villa María, Conociendo que los humedales de Villa María es uno de los pulmones de la ciudad, está siendo destruido por malos ciudadanos que irresponsablemente provocan incendios 
forestales y destruyen el ecosistema por intereses propios. En ese sentido se ha puesto en carpeta el proyecto del Parque Metropolitano que se venía ejecutando en los pantanos de Villa María, por falta de responsabilidad el proyecto que se inició hace 20 años se dejó de lado por las autoridades de turno, retomar este proyecto beneficiaría a toda la población debido que es un área de cuatro mil hectáreas, pero para el parque solo se tomaría 680 hectáreas.

Así mismo, la Municipalidad del Santa a través de la Ley N. ${ }^{\circ} 28245$ Ley marco del sistema nacional de gestión ambiental del Congreso de la República (2004): en coordinación con el Ministerio del Ambiente, el Organismo de Evaluación y Fiscalización Ambiental (OEFA), el Servicio Nacional Forestal y de Fauna Silvestre (SERFOR) y la Autoridad Nacional del Agua (ANA), para que a través de un proyecto de recuperación se pueda traer de nuevo un punto ecológico saludable en beneficio de los habitantes de Chimbote y Nuevo Chimbote.

\section{Conclusiones}

Existen determinados factores que ocasionan que la Municipalidad Provincial Del Santa no sea eficiente en la ejecución de la normatividad de la responsabilidad ambiental en la protección del medio ambiente, como las injerencia políticas, los presupuestos no aprobados para la elaboración de proyectos, conflictos sociales que intervienen y retrasan las evaluaciones, las empresas que buscan beneficiarse a través de sus propios intereses, también existen proyectos retrasadas por incapacidad de los gobernantes de turno, los programas ambientales rechazados porque no lucran, los intereses propios de elegir qué proyecto se aprueba y que proyecto se rechaza.

La ineficacia en la aplicación de la normatividad de la responsabilidad ambiental trae consigo consecuencias irreparables al medio ambiente, los daños que se originan en los humedales de Villa María a través de los 
incendios forestales y el arrojo de desechos ocasionan que la flora desaparezca y espante a toda la fauna silvestre que llega. Además, la contaminación del mar volviendo el agua colores turbias y oscuras ocasiona la muerte de los peces y otras especies, esto es el reflejo del incumplimiento de la normativa. También la falta de supervisión provoca que la contaminación tenga efectos negativos en la salud de las personas y atente contra el derecho de la salud de las personas.

La Municipalidad Provincial del Santa debe hacer cumplir la normatividad de responsabilidad ambiental, que faculta al gobierno local de realizar todas las acciones necesarias en favor del medio ambiente, debe supervisar y sancionar las malas prácticas en coordinación con las distintas entidades ambientales. La gerencia de gestión ambiental no ha cumplido con ejecutar adecuados proyectos lo que ha provocado efectos negativos como el crecimiento de la contaminación en toda la ciudad y los escasos proyectos logrados que no han sido suficiente para disminuir la contaminación del aire por el $\mathrm{CO}_{2}$ de las fábricas, los afluentes arrojados al mar, los afluentes domésticos y los desechos sólidos en la vía pública.

La gerencia de gestión ambiental no cumple su rol eficientemente en la protección del medio ambiente y una de las razones es que no está facultada para aprobar presupuestos o autorizar que proyectos de gran magnitud, solo es un intermediario entre la Municipalidad y el Ministerio del Ambiente en desarrollar proyectos. Sin embargo; la gerencia de gestión ambiental está facultada para supervisar y sancionar a las empresas que no se rigen a la normativa según el sector que se desarrollan, se necesita un control más rígido para hacer cumplir las normas y sanciones ejemplares a las empresas que no cumplan la normativa, en la ciudad de Chimbote el sector pesquero es la que más contamina. 


\section{Referencias}

Andía, J. (2013). Manual de derecho ambiental. Doctrina - Jurisprudencia

- Legislación. 2ª edición, ISBN: 9786120011232 . Lima, Perú: Librería Editorial El Saber.

Bustamante, L., \& León, K. (2015). Análisis de la normativa ambiental peruana en el manejo de residuos sólidos de la construcción y demolición como producto de la excavación en edificaciones. Tesis. Lima, Perú: Universidad Ricardo Palma. Recuperado de: http://repositorio.urp.edu.pe/handle/URP/2218

Cafferatta, N. (2004). Introducción al derecho ambiental. Primera edición, ISBN: 968-817-682-6. México: Instituto Nacional de Ecología (INESEMARNAT).

Collantes, J., \& Giraldo, M. (2020). Responsabilidad ambiental como estrategia competitiva de las organizaciones. Dictamen Libre, (27), 45-65, e-ISSN: 0124-0099. Recuperado de:

Congreso de la República (2004). Ley N. 28245 - Ley marco del sistema nacional de gestión ambiental. Lima, Perú: El Congreso de la República.

Congreso de la República (2003). Ley N.ํ 27972 - Ley Orgánica de Municipalidades. Lima, Perú: El Congreso de la República.

Congreso de la República (2000). Ley N.ํ 27314 - Ley General de Residuos Sólidos. Lima, Perú: El Congreso de la República; Diario Oficial El Peruano.

Costa, M., Santos, L., \& Angelo, C. (2020). Corporate social and environmental responsibility: do consumers perceive it as being an organizational value?. Gestão \& Produção, 27(4), 1-21, e-ISSN: 1806-9649. Recovered from: https://doi.org/10.1590/0104-530X4688$\underline{20}$

Dehays, J., Baca, L., \& Bosker, J. (2000). Léxico de la Política. México: Fondo 
Artículo Original / Original Article

de Cultura Económica.

Enger, E., \& Smith, B. (2006). Ciencia ambiental. Un estudio de interrelaciones. ISBN: 970-10-5616-7. México: McGraw-Hill / Interamericana Editores, S.A. de C.V.

Femenías, J. (2017). La Culpabilidad en la Responsabilidad por Daño Ambiental y su Relación con el Sistema de Evaluación de Impacto Ambiental. Revista de derecho (Valparaíso), (48), 233-259, e-ISSN: 0718-6851. Recuperado de: http://dx.doi.org/10.4067/S0718$\underline{68512017000100233}$

García, B. (2017). Políticas públicas medio ambientales y la contaminación ambiental en el Mercado "La Paradita", San Jacinto - 2016. Tesis. Nuevo Chimbote, Perú: Universidad César Vallejo.

Gardetti, M. (2004). Creando valor sustentable. Venezuela: Editorial Bussiness \& Sustainability Letter.

MEF (2018). Aceptan Aporte Financiero No Reembolsable que otorga KfW para la preparación de estudio de pre inversión del Proyecto de la Planta de Tratamiento de Aguas Residuales en la ciudad de Chimbote. Resolución Suprema N. ${ }^{\circ}$ 025-2018-EF. Lima, Perú: Ministerio de Economía y Finanzas.

Mohorte, A. (2018). Noruega se ha pasado al coche eléctrico. $Y$ en sus ciudades se respira un aire más limpio que nunca. Ginebra, Suiza: World Economic Forum.

Ministerio del Ambiente (2012). Aprueban el Plan de Recuperación Ambiental de la Bahía EI Ferrol, elaborado por la Comisión Técnica Multisectorial de Alto Nivel, constituida mediante D.S. N. ${ }^{\circ}$ 005-2002PE. Resolución Suprema N. ${ }^{\circ}$ 004-2012-MINAM. Lima, Perú: Diario Oficial El Peruano.

Ministerio del Ambiente (2011). Ley N. ${ }^{\circ} 27446$ - Ley del Sistema Nacional de 
Evaluación de Impacto Ambiental y su Reglamento. Depósito Legal nro. N. ${ }^{\circ}$ 20115817. Lima, Perú: Biblioteca Nacional.

Ministerio Secretaría General de la Presidencia (1994). Ley 19300. Aprueba

Ley Sobre las Bases Generales del Medio ambiente. Chile: Biblioteca Nacional de Chile.

MVCS (2013a,b). Decreto Supremo N..$^{\circ}$ 003-2013-VIVIENDA - Aprueba el Reglamento para la Gestión y Manejo de los Residuos de las Actividades de las Construcción y Demolición. Lima, Perú: Ministerio de Vivienda, Construcción y Saneamiento - VIVIENDA PCM (2004). Aprueban el Reglamento de la Ley N. ${ }^{\circ}$ 27314, Ley General de Residuos Sólidos (24.07.04). Decreto Supremo N. ${ }^{\circ}$ 057-2004-PCM. Lima, Perú: Presidencia del Consejo de Ministros.

Quisocala, A. (2003). Educación Ecología. $2^{a}$ edición. Perú: Titikaka FACEDUC.

Romero, M., Diego, F., \& Álvarez, M. (2006). La contaminación del aire: su repercusión como problema de salud. Revista Cubana de Higiene y Epidemiología, 44(2), 1-14, e-ISSN: 1561-3003. Recuperado de: http://scielo.sld.cu/scielo.php?script=sci arttext\&pid=S1561$\underline{30032006000200008}$

Rojas, C. (2017). Eficacia de las normas de gestión ambiental en empresas pesqueras y la salud de los pobladores de Coishco Viejo, 2017. Tesis. Chimbote, Perú: Universidad César Vallejo.

Sierra, Y. (2018). Perú: Madre de Dios, Huancavelica, Puno y Cusco están expuestas a la contaminación por mercurio. California, Estados Unidos: Mongabay Latam. 


\section{Evelyn Marcia Urquiaga Juárez \\ e-mail: eviurqui@hotmail.com}

Nacida en la ciudad de Chimbote, Provincia Del Santa,

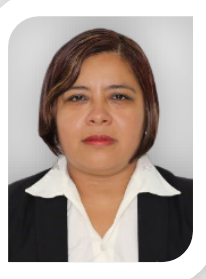

Región Áncash, en Perú, el 3 de octubre del año 1981.

Actualmente laboro en la Universidad Tecnológica del Perú

(UTP), en la Facultad de Derecho y Ciencia Política; mi trayectoria profesional, empieza como servidora del Poder

Judicial de la Corte Superior de Justicia Del Santa; posteriormente me dedique a la defensa libre en el ámbito civil y laboral; en el sector educación; he ejercido cargos administrativos en la Universidad San Pedro (USP); y ejerzo mi tiempo completo a la investigación y docencia universitaria en diferentes instituciones educativas. 